\title{
Influence of the radius of the quantum dot and the nonquilibrium degree on the conversion efficiency of light emitting diodes
}

\author{
Kh. B. Ashurov, M. M. Adilov, S. E. Maksimov, B. L. Oksengendler \\ Institute of Ion-Plasma and Laser Technologies, Uzbek Academy of Sciences, \\ Tashkent, Uzbekistan \\ oksengendlerbl@yandex.ru
}

PACS 75.50.Tt, 81.16.Hc, 87.85.Rs $\quad$ DOI 10.17586/2220-8054-2015-6-6-833-836

\begin{abstract}
We describe the features of the conversion of electrical current into light in light diodes based on an organic matrix containing semiconductor quantum dots. It is shown that the relaxation channels of nonequilibrium electron-hole plasma injected into the quantum dot of the current density through the device. The conversion efficiency increases significantly with a decrease in the radius of the quantum dot.
\end{abstract}

Keywords: light diode, quantum dot, nonequilibrium e-h-plasma, internal efficiency.

Received: 1 November 2015

\section{Introduction}

The problem of converting electrical energy into light has remained an attractive topic for many years [1,2]. Due to the ongoing progress in modern nanotechnology, that problem has become crucially important. From a functional viewpoint, it is also caused by the fact that the electronic spectra of nanoparticles have spectral features both atomic and solid-state structures. On the other hand, the transition to highly non-equilibrium systems is of important for the generation of light from electricity. The possibility of such combination can be estimated for light-emitting diodes (LEDs), having the polymers as the matrix and the quantum dots as the light-converting objects.

\section{Relaxation processes in quantum dot}

The possibility of increasing of the conversion efficiency of the electric current into light can be analyzed on the structure presented in Fig. 1.

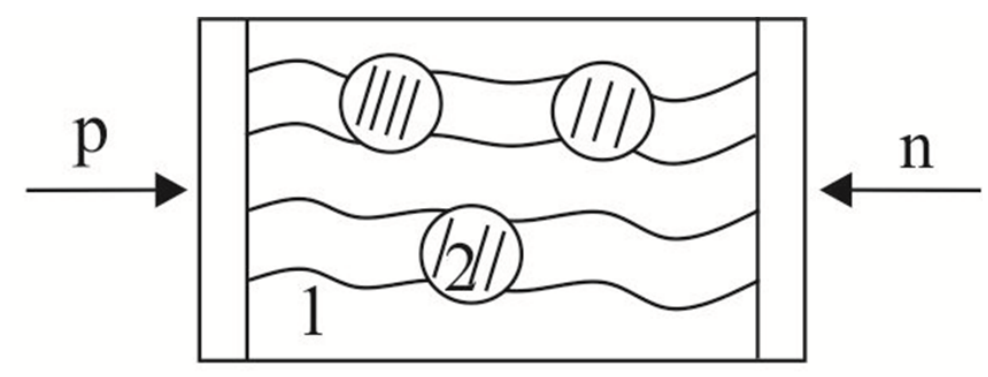

FIG. 1. Schematic diagram of the LEDs on the quantum dots embedded in a polymer matrix ( 1 is the polymer matrix, 2 is the quantum dot) 
Let us assume that the electrons and holes are injected into a quantum dot using a double injection method. Such plasma of e-h pairs relaxes through the three processes: the recombination of electrons and holes by birth of phonons (the probability of relaxation is $\left.1 / \tau_{1}\right)$, recombination by the Auger decay $\left(1 / \tau_{2}\right)$ and the recombination by emission of a photon $\left(1 / \tau_{2}\right)$ (Fig.2). Earlier analysis showed [3], that the first process is less effective than the other ones, so that the internal efficiency for conversion of the current into the light can be written as [4]:

$$
\eta_{\text {intern }}=\frac{1 / \tau_{3}}{1 / \tau_{3}+1 / \tau_{2}} .
$$

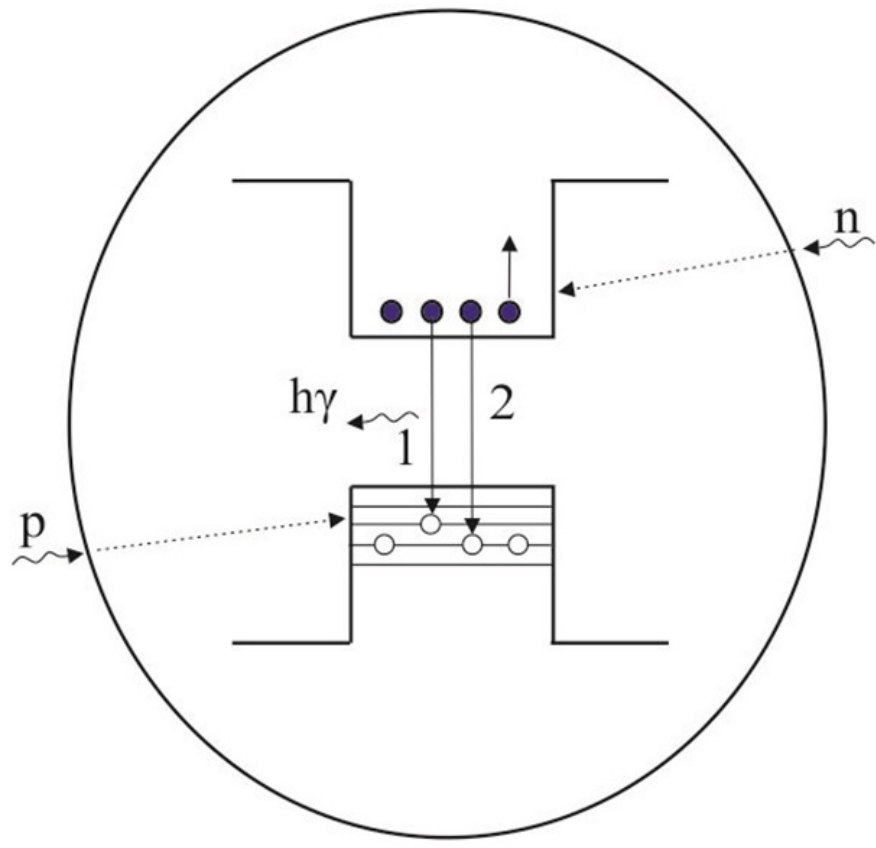

FIG. 2. Recombination processes in quantum dot after injection of electrons $(\mathrm{n})$ into the conduction band and holes $(\mathrm{p})$ into the valence band

It is important to note that the second and third processes, and hence the probabilities $1 / \tau_{2}$ and $1 / \tau_{3}$ have the special features in quantum dots, which are fundamentally different from their behaviors in atoms and in bulk materials. In the following, we will discuss them separately.

\section{Auger recombination}

Quantum dot-based Auger relaxation has been discussed in [5]. The matrix element $M_{i f}$, whose squared value is proportional to the probability $1 / \tau_{2}$ of the process, can be written as $[6]$ :

$$
M_{i f}=\int \psi_{f}^{*}(1) \varphi_{f}^{*}(2) \frac{e^{2}}{r_{12}} \exp \left(-\lambda r_{12}\right) \chi_{i}(1) \phi(2) d^{3} r_{1} d^{3} r_{2}
$$

Here, "i" and "f" means the initial and final states of the wave function of two electrons, respectively, $r_{12}$ is the distance between the electrons, $\lambda^{2}=\left(4 \pi e^{2} \bar{n} / k T\right)$ is the square of the inverse radius of the Debye screening, $\bar{n}$ is the plasma density. 
Calculation of the probability by the method developed in $[6,7]$ allows one to obtain an interesting result, which includes the approach of the atom and the approach of the volume semiconductor as the particular special cases:

$$
1 / \tau_{3}=\frac{\gamma S \bar{n}^{2} A}{\left(\lambda^{2}+B\right)^{4}}
$$

Here, $A$ and $B$ are constants depending on the dielectric constant and the width of the electron band gap of quantum dot. We assume that $S=S_{0} R^{3}, \bar{n}=Z_{\max } / R^{3}$, and $Z_{\max }=j \tau R^{2}$, where $j$ is the current density through the device, $\tau$ is the lifetime of the e-h-plasma, $S_{0}$ is the constant (see. [5]), $Z_{\max }$ is the maximum number of e-h-pairs injected into the quantum dot. Then, the probability of Auger recombination will depend on the specific density of the current through the device and on the radius $R$ of the quantum dot:

$$
1 / \tau_{3}=\gamma S_{0} R^{3} \frac{\bar{n}^{2} \tilde{A}}{(\bar{n}+\tilde{B})^{4}}=\gamma S_{0} R^{3} \frac{\tilde{A}[j \tau / R]^{2}}{[\tilde{B}+j \tau / R]^{4}} .
$$

Here, $\tilde{A}$ and $\tilde{B}$ are the renormalized parameters $A$ and $B$. It is clear from (4) that at low current densities and large quantum dot radii of the (at $j \tau / R<\tilde{\mathbf{B}}$ ) we have:

$$
1 / \tau_{3}=\gamma S_{0} \frac{\tilde{A} \tau}{\tilde{B}^{4}} j^{2} R
$$

i.e. the probability of non-radiative Auger recombination increases rapidly with increased current density and weaker with increased quantum dot radii. At high current densities and small quantum dot radii, the situation is changing fundamentally:

$$
1 / \tau_{3}=\gamma S_{0} \tilde{A} \frac{R^{5}}{j^{2} \tau^{2}}
$$

Thus, the probability of Auger recombination is reduced to smaller $R$ and high densities.

\section{Radiative recombination}

The probability of radiative recombination (the spontaneous emission of a photon in the transition of the electron from a higher state to a lower one) is very different for the atom and for the bulk semiconductor. Namely, the first probability is many times larger than the second one. Taking into account that the quantum dot is an "intermediate object" between the atom and the bulk semiconductor, we consider the following situation. A system of quantum dots with the captured nonequilibrium $e-h$-plasma is similar to that of the Thomson atom model, in which the electrons move in quasi-atomic orbits (see. [8]) on the spread background of positive charge $Z_{\max }$, with an average density $Z_{\max } / R^{3}$. If the orbit of the excited $Z\left(1 \leqslant Z \leqslant Z_{\max }\right)$, then the probability for spontaneous emission of a photon can be written as (in atomic units) [9]:

$$
1 / \tau_{3}=\frac{Z^{4}}{c^{3}}
$$

where $\mathrm{c} \approx 137$ is the speed of light in atomic units.

We note that the value of $Z$ is concentrated to the center of the electron orbit of radius $a_{0}$, with the random probability determined by the Poisson distribution:

$$
\bar{Z}^{Z} e^{-\bar{Z}} / Z \text { ! }
$$


Here, $\bar{Z}=\bar{Z}_{\max } a_{0}^{3} / R^{3}$ is the average charge run by the electron with the radius of orbit $a_{0}$.

\section{The internal conversion efficiency of current into light in a quantum dot}

We determine the value of the boundary charge from the relation $1 / \tau_{2}=1 / \tau_{3}$. Obviously, at $Z>Z^{*}=\left[\tau_{a t} \gamma s_{0} c^{3} \tilde{A} / \tau^{2}\left(R^{5} / j^{2}\right)\right]^{1 / 4}$, the fluorescence probability is greater than that for Auger recombination, and the partial internal efficiency can be written as:

$$
\eta_{\text {intern }}(Z)=1-\frac{\left(1 / \tau_{2}\right)}{\left(1 / \tau_{3}\right)}=1-\frac{D\left(R^{5} / j^{2}\right)}{Z^{4}}
$$

We imply here a strong non-equilibrium (large $j$ values) and the small quantum dot radius size $(\mathrm{R}) ; D=\gamma s_{0} \tilde{A} c^{3} \tau_{a t}, \tau_{a t}$ is the characteristic atomic time.

The value $\eta_{\text {intern }}(Z)$ must be averaged over all $Z \geqslant Z^{*}$; we do this using not the Poisson distribution, but with a normal Gaussian distribution:

$$
\bar{\eta}_{\text {intern }} \geqslant \int_{Z^{*}}^{\infty} \frac{1}{\sqrt{2 \pi} \sigma} \exp \left[-\frac{(Z-\bar{Z})}{2 \sigma^{2}}\right] d Z-\int_{Z^{*}}^{\infty} \frac{D\left(R^{5} / j^{2}\right)}{Z^{4}} \frac{1}{\sqrt{2 \pi} \sigma} \exp \left[-\frac{(Z-\bar{Z})}{2 \sigma^{2}}\right] d Z,
$$

where $\sigma=\sqrt{Z_{\max }\left(\frac{a_{0}}{R}\right)^{3}\left(1-\frac{a_{0}}{R}\right)^{3}}$,

$$
\bar{Z}=Z_{\max }\left(a_{0} / R\right)^{3}, \quad Z^{*}=\left[D\left(R^{5} / j^{2}\right)\right]^{1 / 3} .
$$

It is clear that the main contribution comes from the first term of Eq. (10). This shows that $\bar{\eta}_{\text {intern }}$ increases as $Z^{*}$ decreases, i.e. the efficiency of conversion of current energy into light increases with decreased quantum dot size.

\section{Acknowledgments}

This work is supported by the Committee for Coordination of Science and Technology Development under Cabinet of Ministers of the Republic of Uzbekistan (contract F3-FAF158).

\section{References}

[1] Berg A., Dean P. Light-emitting diodes. Moscow, Mir, 1979, 686 p. (in Russian).

[2] Pope M., Swenberg Ch. Electronic Processes in Organic Crystals. Clarendon Press, Oxford University Press, New York, 1982, V.1 (543 p.), V.2. (462 p.).

[3] Ridley B. Quantum Processes in Semiconductors. University of Essex, Clarendon Press, Oxford, 1982, $304 \mathrm{p}$.

[4] Vikulin I.M., Stafeev V.I. Physics of Semiconductor Devices. Moscow, Radio and communication, 1990, 264 p. (in Russian).

[5] Klimov V.I. Nanocrystal Quantum Dots: From fundamental photophysics to multicolor lasing. Los Alamos Science, 2003, 28, P. 214-220.

[6] Parilis E.S., Effekt Ozhe (Auger Effect), Tashkent, Fan, 1969, 211 p. (in Russian).

[7] Landsberg P.T. Recombination in Semiconductors. Cambridge University Press, Cambridge, 1991, 595 p.

[8] Shpolsky E.V. Atomic physics. V.1. Moscow, Science, 1984. (in Russian).

[9] Migdal A.B. Qualitative methods in quantum theory. Moscow, Science, 1975. (in Russian). 